\title{
Neutrino cross-section measurement prospects with SBND
}

\author{
Nicola McConkey* for the SBND Collaboration \\ University of Sheffield \\ E-mail: n.mcconkeyesheffield.ac.uk
}

\begin{abstract}
SBND (Short-Baseline Near Detector) is a 112 ton liquid argon time projection chamber (LArTPC) neutrino detector under construction in the Fermilab Booster Neutrino Beam. Together with MicroBooNE and ICARUS-T600 detectors, SBND will search for short baseline neutrino oscillations in the $1 \mathrm{eV}^{2}$ mass range. SBND will also perform detailed studies of the physics of neutrino-argon interactions, thanks to a data sample of millions of electron and muon neutrino interactions. Finally SBND plays an important role in the on-going R\&D effort to develop the LArTPC technology, testing several technologies that can be used in a future kiloton-scale neutrino detectors for a long-baseline experiment. We will discuss the detector design, its current status, and the physics program, with a particular focus on the neutrino cross-section measurement prospects.
\end{abstract}

The 19th International Workshop on Neutrinos from Accelerators-NUFACT2017

25-30 September, 2017

Uppsala University, Uppsala, Sweden

${ }^{*}$ Speaker. 
Table 1: Parameters of the three SBN detectors.

\begin{tabular}{|l|c|c|}
\hline Detector & Baseline (m) & Active LAr mass (tonnes) \\
\hline SBND & 110 & 112 \\
MicroBooNE & 470 & 87 \\
ICARUS T-600 & 600 & 476 \\
\hline
\end{tabular}

\section{Introduction}

Over the past 20 years there have been several experimental anomalies observed in neutrino experiments with baselines of the order of $<1 \mathrm{~km}$, which are referred to as short baseline experiments. Most notably the Liquid Scintillator Neutrino Detector (LSND) [1], which was a decay at rest experiment measuring antineutrino oscillations from a decay-at-rest $\bar{v}_{\mu}$ source, observed a $3.8 \sigma$ excess in the $\bar{v}_{e}$ appearance channel, as is shown in figure 1. Subsequent measurements made by MiniBooNE [2] in the Booster Neutrino Beam (BNB) at Fermilab showed a $3.4 \sigma$ excess in $v_{\mu}>v_{e}$ channel and a $2.4 \sigma$ excess in the $\bar{v}_{\mu-}>\bar{v}_{e}$ channel.

These oscillation excesses can be interpreted as a large $\Delta \mathrm{m}^{2}$ oscilllation to an additional "sterile" neutrino state, which does not interact via the weak interaction. This $\Delta \mathrm{m}_{14}^{2}$ is around $1 \mathrm{eV}$.

The Short Baseline Neutrino (SBN) Program is a three detector neutrino measurement programme in the Booster Neutrino Beam (BNB) at Fermilab [3]. The three detectors, with parameters as described in table 1 are functionally identical Liquid Argon Time Projection Chambers (LArTPC). The use of the same nuclear target and detector technology reduce the effect of systematic uncertainties across the three detector system.

The primary physics goal of the SBN programme is to measure neutrino oscillations in the BNB beam, to definitively confirm or refute the results of LSND and MicroBooNE at the $5 \sigma$ level. This experiment will measure both electron neutrino appearance and muon neutrino disappearance. The sensitivity plots for SBN are shown in figure 1.

SBND is the near detector for the SBND programme and will measure the unoscillated neu-
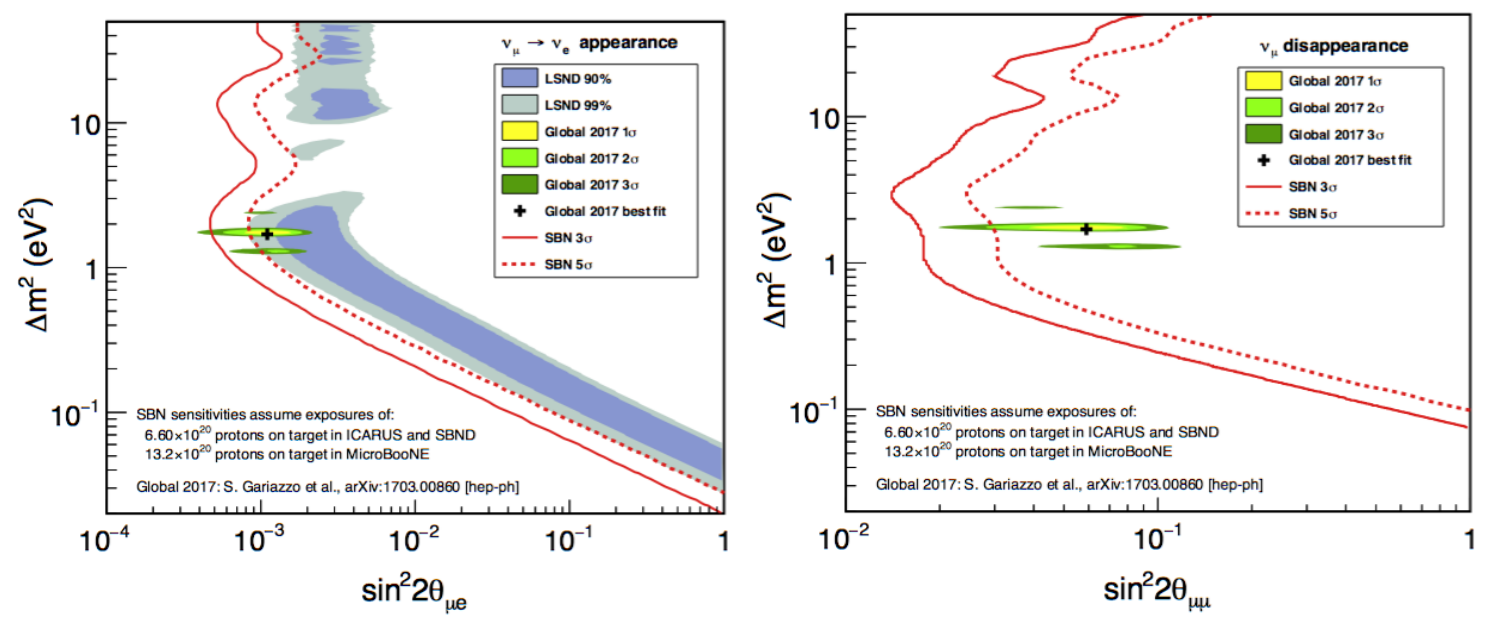

Figure 1: The electron neutrino appearance (left) and muon neutrino disappearance (right) sensitivities of the SBN program, in the context of the LSND measured values and global best fit from [4]. 
trino flux. SBND will have extremely high statistics for $v_{\mu}$ and $v_{e}$ charged current and neutral current interactions, and will be used to tune the flux and cross-section models to produce unoscillated predictions for the two far detectors MicroBooNE and ICARUS.

In addition to providing near detector measurements for the SBN Program, the SBND detector allows us to study neutrino-nucleus interactions in argon with unprecedented sensitivity.

The BNB is a low energy neutrino beam at Fermilab, produced from $8 \mathrm{GeV}$ protons on a beryllium target. The peak neutrino energy is at $700 \mathrm{MeV}$, and the full spectrum is shown in figure 2. It is a muon neutrino beam with electron neutrino contamination of less than $0.5 \%$, which is well characterised and has been stably running for over a decade; the SBN detectors sit in the same beam as the MiniBooNE detector.

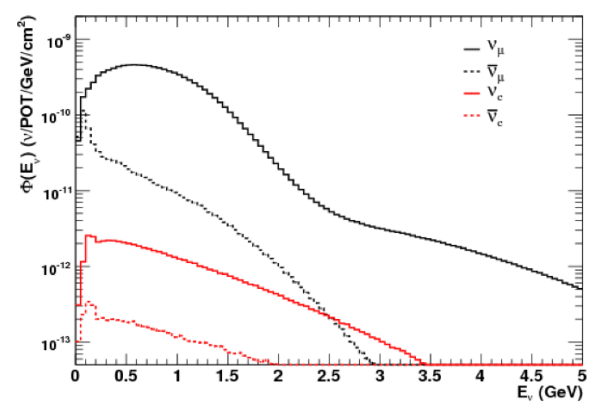

Figure 2: The Booster Neutrino Beam energy spectrum.

\section{SBND Detector Design and current status}

SBND is a LAr TPC with charge and light readout. It has an active volume of $5 \mathrm{~m} \times 4 \mathrm{~m} \times 4 \mathrm{~m}$, composed of two drift regions, with a central cathode, and two wire readout planes, as shown in figure 3. The drift direction is perpendicular to the neutrino beam direction. Each wire readout plane, or Anode Plane Assembly (APA) consists of two interconnected steel APA frames, which support 3 planes of copper-beryllium wires at pitch and plane spacing of $3 \mathrm{~mm}$. Figure 3 shows the orientation and layout of these wire planes: a vertical collection plane (Y), and two induction planes (U,V) at $\pm 60^{\circ}$ angles to the vertical. The signal is collected at the outer edge of the APA, and there is a jumpered interconnect between the two APA frames.

The central cathode (CPA) is a welded, electropolished assembly composed of stainless steel frames supporting mesh frames. Between the layers of conductive mesh, wavelength shifter coated reflector foils are sandwiched, which enhance the uniformity of light collection in SBND. The cathode will be biased at $-100 \mathrm{kV}$, with a coaxial high voltage feedthrough composed of stainless steel core and grounding sheath with polyethylene (PE) insulator bringing this bias in and contacting the cathode donut with a spring-loaded tip.

The SBND field cage is composed of roll formed aluminium profiles, with PE end caps. This is identical to the field cage material which will be used in ProtoDUNE [5] and DUNE [6].

SBND has a composite photon detector system, the baseline of which is an array of 8" Hammamatsu R5912 photomultiplier tubes (PMT), coated in wavelength shifting tetraphenyl butadeine 

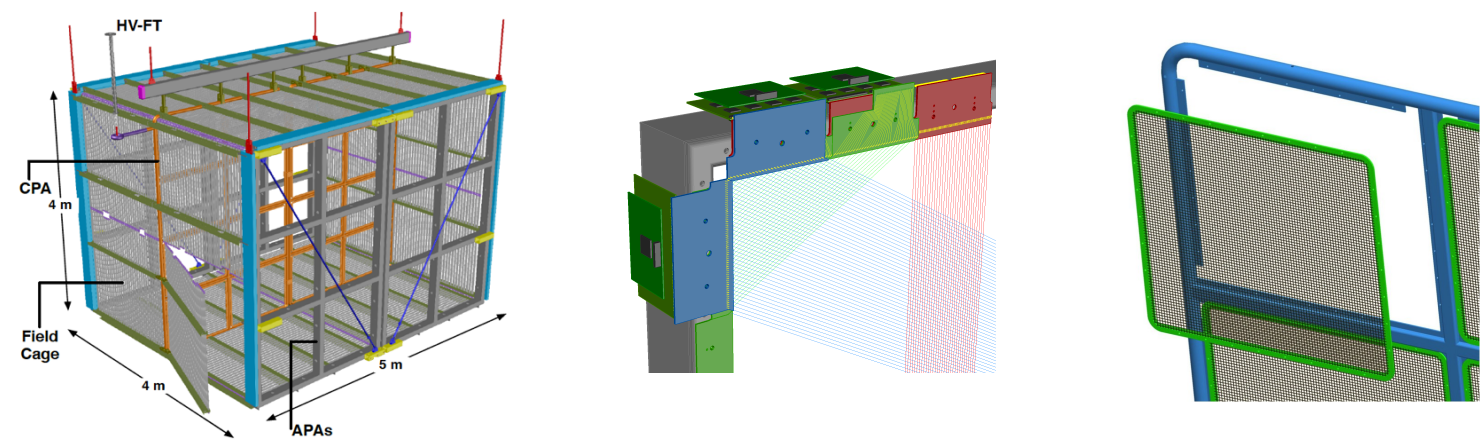

Figure 3: Left: The SBND TPC. Centre: APA wire planes. Right: CPA mesh panel and frame.

(TPB). In addition to this system, there are two other proposed technologies for light collection in SBND: the ARAPUCA and the light guide bars. The ARAPUCA is a novel photon collection device which is composed of dichroic filters on a highly internally reflective box, read out by a Silicon PhotoMultiplier (SiPM) [7]. The light guide bars are composed of TPB coated acrylic strips, read out by an array of SiPMs [8]. This composite light detector system is mounted behind each wire readout plane, as shown in figure 4 . The photon detection system is both a test-bed for new technology, and an excellent calorimetric tool with fast timing capabilities.

As SBND is located at the surface with only a few metres concrete overburden, and in order to mitigate the cosmic ray background in the detector, it is surrounded by seven planes of extruded scintillator strips read out by MPPC, which act as a cosmic ray tagger, as is shown in figure 4 [9].
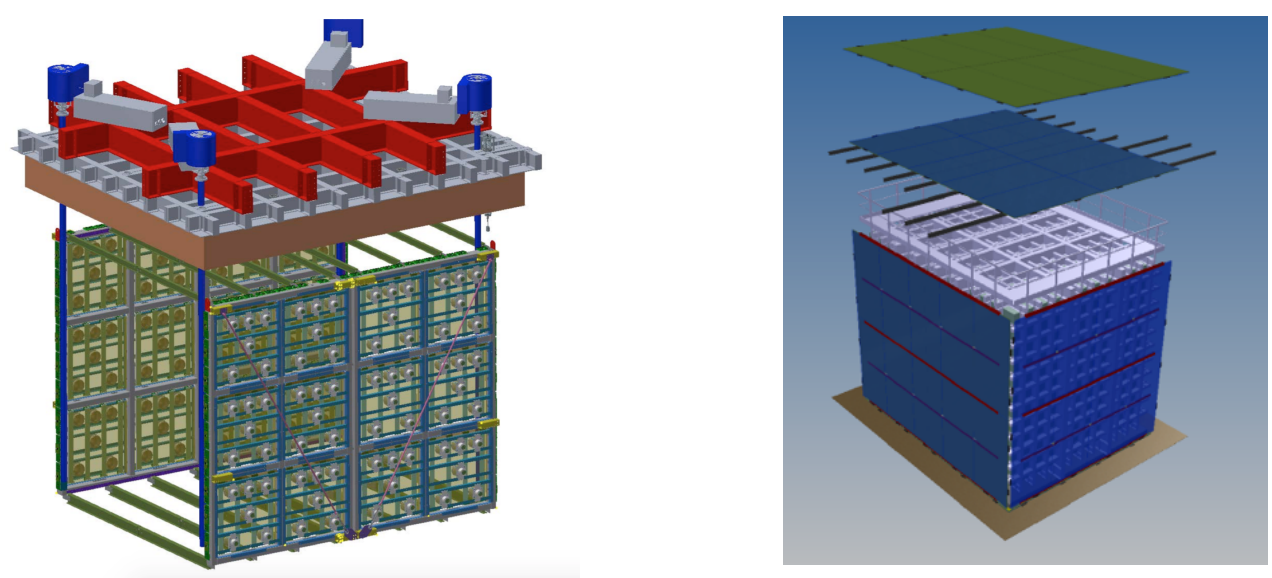

Figure 4: Left: The SBND photon detection system mounted behind the APAs. Right: Cosmic ray tagger system surrounding the SBND cryostat.

The SBND LAr TPC gives full 3D imaging with millimetre granularity, allowing for precise measurement of topological and calorimetric information.

\section{Neutrino cross section measurements}

Since SBND is situated at a distance of $110 \mathrm{~m}$ from the target, it has an extremely high event rate for neutrino interactions. Combined with the excellent granularity of the detector, this makes 
SBND an extremely powerful detector for measuring interaction properties of the neutrino on argon nuclei.

The estimated number of events in the SBND active volume for a $6.6 \times 10^{20}$ POT, from GENIE simulations as detailed in [3], gives 7 million $v_{\mu}$ events and 50,000 $v_{e}$ events in 3 years. To put this in context of other LAr $v$ detectors, SBND will collect the entire MicroBooNE data set in just 2 months. This will quickly reduce the statistical uncertainty to well below the percent level, making systematic errors dominant.

With high statistics, detector granularity, and good particle identification, the study of exclusive neutrino interaction topologies is possible. At SBND, Charged Current (CC) interactions with no pions in the final state $(0 \pi)$ are the dominant channel, which can be used to quantify nuclear effects in $v$-Ar scattering. SBND will carry out a direct experimental investigation and quantification of nuclear effects, measuring the impacts of interactions with the argon nucleus on rates, final states and kinematics. This data will inform neutrino monte-carlo generators, and aid in disentangling neutrino-nuclear interaction phenomenology by discriminating between final state interaction models. One example of the statistical power of this is in measurement of correlated nucleon pairs; there will be 360,000 events per year with $\mu+2 p$ in the final state.

The high interaction rate allows SBND to measure interaction channels which remain unmeasured on argon. There are many rare interaction channels which can be probed by SBND, for example production of hyperons $\Lambda^{0}$ and $\Sigma^{+}$, for which SBND will collect a total data set of several thousand events over 3 years, more than the current historical data set in one month.

In addition to the large number of $v_{\mu}$ events, there will be a high statistics $v_{e}$ sample, which will allow for both inclusive and exclusive measurements for electron neutrino interactions. There will be around 37,000 $v_{e} \mathrm{CC}$ events over the 3 year run. Measurements of this interaction will be extremely beneficial for both SBN and DUNE physics programmes.

The BNB neutrino flux spectrum at SBND covers the neutrino energy at both the first and second oscillation maxima for DUNE, at energies of $2.6 \mathrm{MeV}$ and $0.8 \mathrm{MeV}$ respectively. This means there will be a high statistics sample of neutrino interactions at energies relevant to long baseline oscillation measurements in argon.

Since SBND has a high event rate of the unoscillated BNB, it is in an ideal position to perform the neutrino beam flux normalisation for the SBN experiment. The elastic scattering of neutrinos on electrons is a well known cross section, and will provide a unique topological signature in the LAr TPC: a very forward electron with no activity around the vertex, as is shown in figure 5. There will be around 300 events expected in the 3 year data run, and this will allow SBND to normalise the flux for the neutrino oscillation measurements.

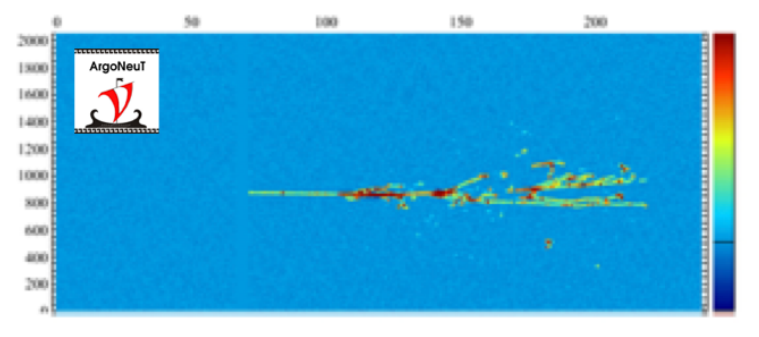

Figure 5: An neutrino-electron elastic scattering event recorded at ArgoNeut [10]. 


\section{Current status and future timeline}

The SBND detector is currently in the advanced stages of component construction. The APA frames have been manufactured, machined, assembled and measured to be flat to the specification tolerance of $\pm 0.5 \mathrm{~mm}$. After significant prototyping and development, APA two winding systems, one manual, one semi-automated, are operational, as shown in figure 6 and the first APA frames are being wired. The CPA frames have been welded and test fitted, as shown in figure 7, and the mesh frames are under construction. Field cage components are ready for testing and assembly.
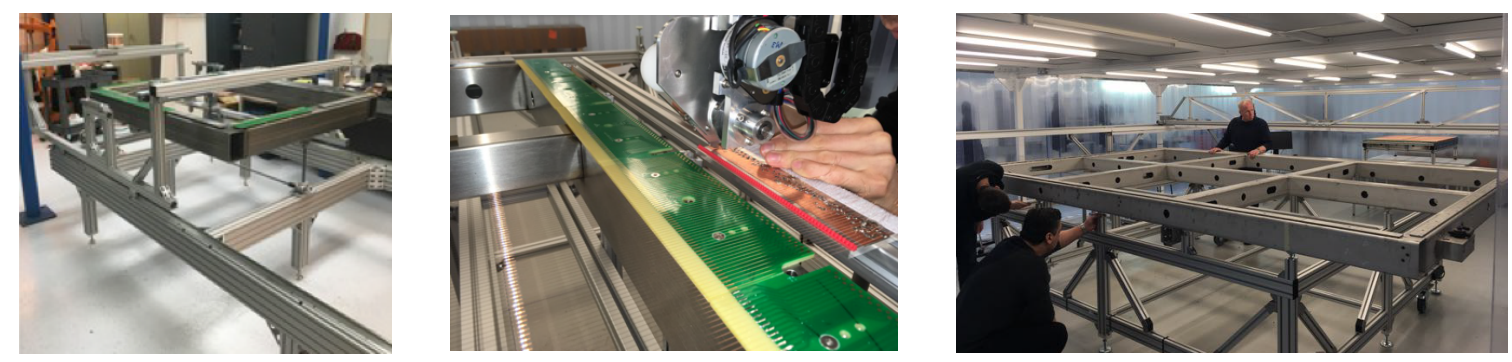

Figure 6: Left: APA prototype manual winding machine with prototype frame. Centre: Semi-automated APA winding machine. Right: APA frame preparation on the semi-automated winding machine table.

With regards to the photon detection system, the baseline system of PMTs is in production, the light guide bar system prototyping is complete and ready to manufacture, and the ARAPUCA devices are in the advanced stages of development.

The cryo-testing vessel, shown in figure 7, is commissioned and ready to use for testing wired APAs. The cosmic ray tagger has been constructed, deployed and operated in the SBND pit, which can be seen in figure 7 .
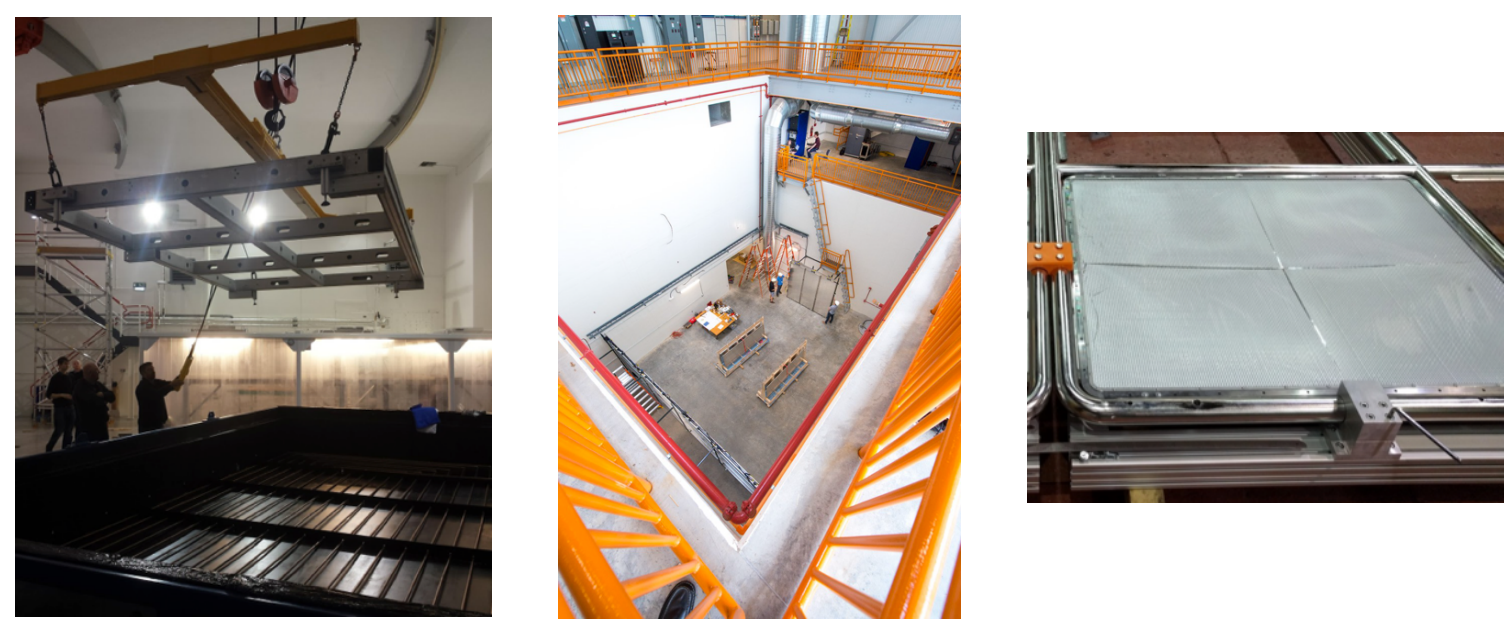

Figure 7: Left: An APA frame is lowered into a cryogenic test vessel for commissioning. Centre: The SBND pit, with cosmic ray tagger panels taking data. Right: Trial fitting of a CPA panel.

The components will be shipped to Fermilab, where detector assembly will take place in 2018. The detector will be commissioned and installed in the pit in 2019, with the first neutrino data expected soon after. 


\section{Summary and Conclusions}

SBND is the near detector for the SBN programme, measuring the unoscillated BNB in order to make systematic and flux constraints for a sterile neutrino search. SBND will measure neutrinoargon interactions with unprecedented sensitivity due to excellent detector characteristics and high event rates. This has the potential to transform our understanding of low energy $v$-Ar interactions.

SBND is currently under construction, and will begin taking neutrino data in 2019.

\section{References}

[1] A. Aguilar-Arevalo et al. [LSND Collaboration], Phys. Rev. D 64 (2001) 112007 doi:10.1103/PhysRevD.64.112007 [hep-ex/0104049].

[2] A. A. Aguilar-Arevalo et al. [MiniBooNE Collaboration], Phys. Rev. Lett. 110 (2013) 161801 doi:10.1103/PhysRevLett.110.161801 [arXiv:1303.2588 [hep-ex]].

[3] M. Antonello et al. [MicroBooNE and LAr1-ND and ICARUS-WA104 Collaborations], arXiv:1503.01520 [physics.ins-det].

[4] S. Gariazzo, C. Giunti, M. Laveder and Y. F. Li, JHEP 1706 (2017) 135 doi:10.1007/JHEP06(2017)135 [arXiv:1703.00860 [hep-ph]].

[5] B. Abi et al. [DUNE Collaboration], arXiv:1706.07081 [physics.ins-det].

[6] R. Acciarri et al. [DUNE Collaboration], arXiv:1512.06148 [physics.ins-det].

[7] A. A. Machado and E. Segreto, JINST 11 (2016) no.02, C02004. doi:10.1088/1748-0221/11/02/C02004

[8] Z. Moss, J. Moon, L. Bugel, J. M. Conrad, K. Sachdev, M. Toups and T. Wongjirad, arXiv:1604.03103 [physics.ins-det].

[9] M. Auger et al., Instruments 1 (2017) no.1, 2 doi:10.3390/instruments1010002 [arXiv:1612.04614 [physics.ins-det]].

[10] C. Anderson et al., JINST 7 (2012) P10019 doi:10.1088/1748-0221/7/10/P10019 [arXiv:1205.6747 [physics.ins-det]]. 